\title{
Entschließung der Konferenz der Informationsfreiheitsbeauftragten vom 2. Dezember 2016
}

Unter dem Vorsitz der Landesbeauftragten für Datenschutz und Informationsfreiheit Nordrhein-Westfalen, Helga Block, hat die Konferenz der Informationsfreiheitsbeauftragten in Deutschland am 2. Dezember 2016 in Düsseldorf getagt.

Mit der Entschließung „Nicht bei Open Data stehenbleiben: Jetzt auch Transparenzgesetze in Bund und Ländern schaffen!“ wiederholen und verstärken die Informationsfreiheitsbeauftragten ihre Forderung nach umfassenden Transparenzgesetzen.

Individuelle Informationszugangsansprüche zu amtlichen Informationen gibt es noch immer nicht in allen Ländern. Auch dort, wo sie geregelt sind, ist es für die Bürgerinnen und Bürger zum Teil schwierig, sich über ihre Rechte zu informieren. Hinzu kommt, dass auch die Regelungen zur antragsunabhängigen Veröffentlichung von amtlichen Daten durch öffentliche Stellen noch unzureichend sind.

Am 14. Oktober 2016 haben Bund und Länder beschlossen, Open Data-Gesetze mit vergleichbaren Standards zu erlassen. Zwar begrüßen die Informationsfreiheitsbeauftragten diese Zielrichtung. In wesentlichen Punkten greift der Beschluss jedoch zu kurz. Die Verwaltung wird nicht allein dadurch transparent, dass sie Rohdaten veröffentlicht. Auch darüberhinausgehende amtliche Informationen wie etwa Konzepte, Verträge und Aktenpläne sind zu veröffentlichen. Und die aktive Veröffentlichung muss verpflichtend sein.

Die bekanntgewordenen Pläne des Bundes zur Umsetzungen des Beschlusses genügen diesen Anforderungen nicht.

Bund und Länder sollten den Beschluss der Regierungschefs umsetzen, indem sie Open Data-Regelungen in Transparenzgesetze aufnehmen. Länder, die noch nicht über solche Gesetze verfügen, sollten nach Auffassung der Informationsfreiheitsbeauftragten vorhandene Informationsfreiheitsgesetze entsprechend fortentwickeln. Auch fordert die Konferenz jene Länder auf, die keinen allgemeinen Anspruch auf Informationszugang gewähren, endlich ein modernes Informationsrecht einzuführen.

\section{Entschließung}

„Nicht bei Open Data stehenbleiben: Jetzt auch Transparenzgesetze in Bund und Ländern schaffen! "

Die Konferenz der Informationsfreiheitsbeauftragten fordert die Gesetzgeber in Bund und Ländern auf, jetzt flächendeckend

1 Bei Enthaltung des Bundes
Transparenzgesetze zu schaffen. Solche Gesetze verbinden den individuellen, antragsgebundenen Informationszugangsanspruch mit der Verpflichtung öffentlicher Stellen, bestimmte Informationen aktiv auf Informationsplattformen im Internet zu veröffentlichen.

Anlass für die Forderung ist ein Beschluss der Regierungschefs von Bund und Ländern vom 14. Oktober 2016. Nach dieser Vereinbarung werden Bund und Länder Open-Data-Gesetze erlassen und das Ziel verfolgen, bundesweit vergleichbare Standards für den Zugang zu öffentlichen Datenpools zu erreichen.

Die Informationsfreiheitsbeauftragten befürworten zwar die Zielrichtung des Beschlusses; dieser greift jedoch zu kurz. Neben der Bereitstellung von Rohdaten in standardisierten und offenen Formaten für eine Weiterverwendung gebietet die Transparenz öffentlichen Handelns, zusammenhängende, aus sich heraus nachvollziehbare Unterlagen zur Verfügung zu stellen. Hierfür kommen beispielsweise Verträge, Gutachten, Studien, umweltrelevante Konzepte, Pläne, Programme oder Zulassungsentscheidungen, Berichte, Protokolle, Beschlüsse, Organisationserlasse, Statistiken, öffentliche Planungen, Haushalts-, Stellen-, Organisations-, Geschäftsverteilungs- und Aktenpläne, Drucksachen, Verwaltungsvorschriften oder wesentliche Bestandteile von Subventions- und Zuwendungsvergaben und Baugenehmigungen sowie die wesentlichen Unternehmensdaten öffentlicher Beteiligungen einschließlich der Vergütung der Leitungsebenen infrage.

Daher fordert die Konferenz, dass Bund und Länder ihre Behörden verpflichten, derartige Dokumente grundsätzlich im Internet zu veröffentlichen. Der bekannt gewordene Entwurf des Eckpunktepapiers des Bundes vom 18.10.2016² genügt diesen Anforderungen nicht. Anstatt separate Gesetze zu schaffen oder die Regelungen den eher informationstechnisch orientierten E-Government-Gesetzen zu überlassen, sollte der Beschluss der Regierungschefs von Bund und Ländern so umgesetzt werden, dass Open-Data-Regelungen in Transparenz-gesetze aufgenommen werden. Länder, die noch nicht über solche Gesetze verfügen, sollten nach Auffassung der Informationsfreiheitsbeauftragten vorhandene Informationsfreiheitsgesetze entsprechend fortentwickeln. Auch fordert die Konferenz jene Länder auf, die keinen allgemeinen Anspruch auf Informationszugang gewähren, endlich ein modernes Informationsrecht einzuführen.

2 Siehe netzpolitik.org: Eckpunktepapier aus dem BMI zum ,Open-Data-Gesetz 